\title{
Radiofrequency Ablation of Liver Tumors
}

\author{
Shaunagh McDermott, FFR RCSI ${ }^{1}$ Debra A. Gervais, MD ${ }^{1,2}$ \\ ${ }^{1}$ Division of Abdominal Imaging and Interventional Radiology, \\ Department of Radiology \\ 2 Division of Pediatric Imaging, Department of Radiology, \\ Massachusetts General Hospital, Boston, Massachusetts

\begin{abstract}
Address for correspondence Shaunagh McDermott, FFR RCSI, Division of Abdominal Imaging and Interventional Radiology, Department of Radiology, Massachusetts General Hospital, 55 Fruit St., White 270, Boston, MA 02114 (e-mail: smcdermott1@partners.org).
\end{abstract}

Semin Intervent Radiol 2013;30:49-55

\begin{abstract}
Keywords

- radiofrequency ablation

- liver

- hepatocellular carcinoma

- metastases

- technique

Radiofrequency ablation (RFA) is an alternative therapy for hepatocellular carcinoma and liver metastases when resection cannot be performed or, in the case of hepatocellular carcinoma, when transplant cannot be performed in a timely enough manner to avoid the risk of dropping off the transplant list. RFA has the advantage of being a relatively low-risk minimally invasive procedure used in the treatment of focal liver tumors. This review article discusses the current evidence supporting RFA of liver tumors, as well as the indications, complications, and follow-up algorithms used after RFA.
\end{abstract}

Objectives: Upon completion of this article, the reader will be able to explain the role of radiofrequency ablation in the treatment of primary and secondary tumors of the liver.

Accreditation: This activity has been planned and implemented in accordance with the Essential Areas and policies of the Accreditation Council for Continuing Medical Education through the joint sponsorship of Tufts University School of Medicine (TUSM) and Thieme Medical Publishers, New York. TUSM is accredited by the ACCME to provide continuing medical education for physicians.

Credit: Tufts University School of Medicine designates this journal-based CME activity for a maximum of 1 AMA PRA Category 1 Credit $^{\mathrm{TM}}$. Physicians should claim only the credit commensurate with the extent of their participation in the activity.

Radiofrequency ablation (RFA) is an alternative therapy for hepatocellular carcinoma (HCC) and liver metastases when resection cannot be performed or, in the case of HCC, when transplant cannot be performed in a timely enough manner to avoid the risk of dropping off the transplant list. RFA has the advantage of being a relatively low-risk minimally invasive procedure for the treatment of focal liver tumors. In this review article we discuss the science, complications, and imaging follow-up after RFA, as well as its indications.

\section{Technique}

The goal of RFA is to completely destroy a tumor without damaging the surrounding liver tissue by inducing thermal injury to the tissue through electromagnetic energy deposition. In the most common configuration of clinical RFA, monopolar RFA, the patient is a part of a closed-loop circuit that includes an RF generator, a needle electrode, and a large dispersive electrode (grounding pads). ${ }^{1}$ This creates an alternating electric field within the tissue that causes agitation of the ions present in the target tissue that surrounds the electrode, resulting in frictional heat around the electrode. ${ }^{2}$ The discrepancy between the small surface area of the needle electrode and the large area of the grounding pads causes the generated heat to be focused and concentrated around the needle electrode while the grounding pads disperse the energy over a larger area to avoid skin burns. ${ }^{3}$

Permanent tissue destruction occurs at temperatures of $\geq 45^{\circ} \mathrm{C}$. With temperatures from $46^{\circ} \mathrm{C}$ to $60^{\circ} \mathrm{C}$, irreversible cellular damage is produced only after relatively longer periods of exposure; in contrast, temperatures between $60^{\circ} \mathrm{C}$ and $100^{\circ} \mathrm{C}$ cause almost instantaneous protein coagulation with irreversible damage to mitochondria and cytosolic cell enzymes. When temperatures exceed $100^{\circ} \mathrm{C}$, tissue fluids undergo boiling, vaporization, and ultimately carbonization. ${ }^{3}$ Vaporization, which occurs when tissues are heated to $>100$ to $110^{\circ} \mathrm{C}$,
Issue Theme Liver Malignancies; Guest Editor, Daniel B. Brown, MD, FSIR
Copyright @ 2013 by Thieme Medical Publishers, Inc., 333 Seventh Avenue, New York, NY 10001, USA. Tel: +1(212) 584-4662.
DOI http://dx.doi.org/ 10.1055/s-0033-1333653. ISSN 0739-9529. 
A
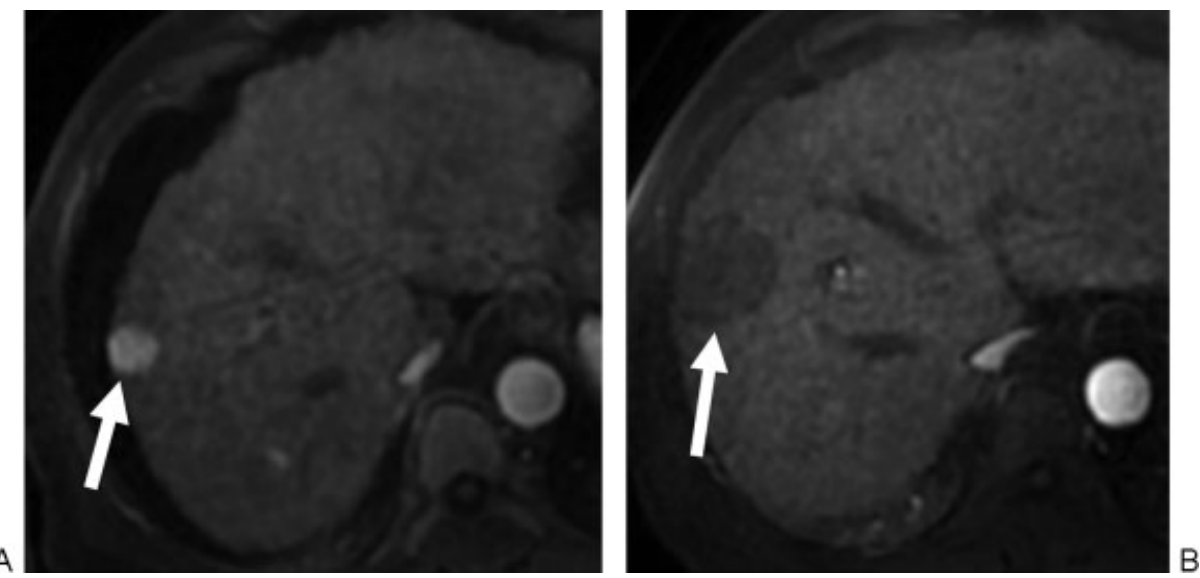

Figure 1 A 74-year-old man with cirrhosis secondary to alcohol abuse. (A) Axial T1 fat-saturated image postgadolinium demonstrates a 1.8-cm arterially enhancing lesion (arrow). (B) One month postablation, axial T1 fat-saturated image demonstrates a zone of ablation (arrow) that is larger than the original tumor with no evidence of residual enhancing tumor.

produces significant gas that both serves as an insulator and retards the ability to effectively establish a RF field. ${ }^{1}$ Therefore, the key aim of RFA is to achieve and maintain a 50 to $100^{\circ} \mathrm{C}$ temperature range throughout the entire target volume.

Another important factor that affects the success of RFA is the ability to ablate all viable tumor tissue and an adequate tumor-free margin. To achieve rates of local tumor recurrence with RFA that are comparable with those obtained by hepatic resection, a 360-degree 1 -cm-thick tumor-free margin around each tumor should be obtained, which may require multiple overlapping ablations. ${ }^{4}$ Thus the target diameter of an ablation must be $2 \mathrm{~cm}$ larger than the diameter of the tumor that undergoes treatment ( $\mathbf{- F i g . 1}$ ). ${ }^{2}$ Inadequate ablation can be due either to heterogeneity of tissue composition, by which differences in tumor density, including fibrosis and calcification, alter electrical and thermal conductance, and blood flow, by which perfusion-mediated tissue cooling (vascular flow) reduces the extent of thermally induced coagulation. ${ }^{1}$

RFA is usually performed following the administration of intravenous sedation and analgesia with standard cardiac, blood pressure, and oxygen monitoring. Targeting of the lesion can be performed with ultrasound, computed tomography (CT), or magnetic resonance imaging (MRI), depending on operator preference and the local availability of dedicated equipment.

\section{Complications}

RFA is a safe procedure with very low rates of death and major complications. Complications include abdominal hemorrhage (-Fig. 2), hepatic abscess, pleural effusion, hepatic infarction, bronchobiliary fistula, bile peritonitis, biloma, hemobilia, thrombosis of vessels in the hepatic venous system, skin burn, tumor seeding, and perforation of the stomach, intestine, or diaphragm.

In a multicenter study by Koda et al on 16,346 treated nodules in 13,283 patients, 579 complications (3.5\%) were observed; five patients (0.04\%) died due to complications of RFA. ${ }^{5}$ A total of 276 hepatic injuries (1.69\%) occurred including 75 liver infarctions (0.469\%) and 32 liver abscesses (0.19\%), 110 bile duct injuries (0.67\%), and 37 bilomas (0.23\%). Other hepatic injuries included portal thrombosis, refractory ascites, and hepatic failure. ${ }^{5}$ Another study found
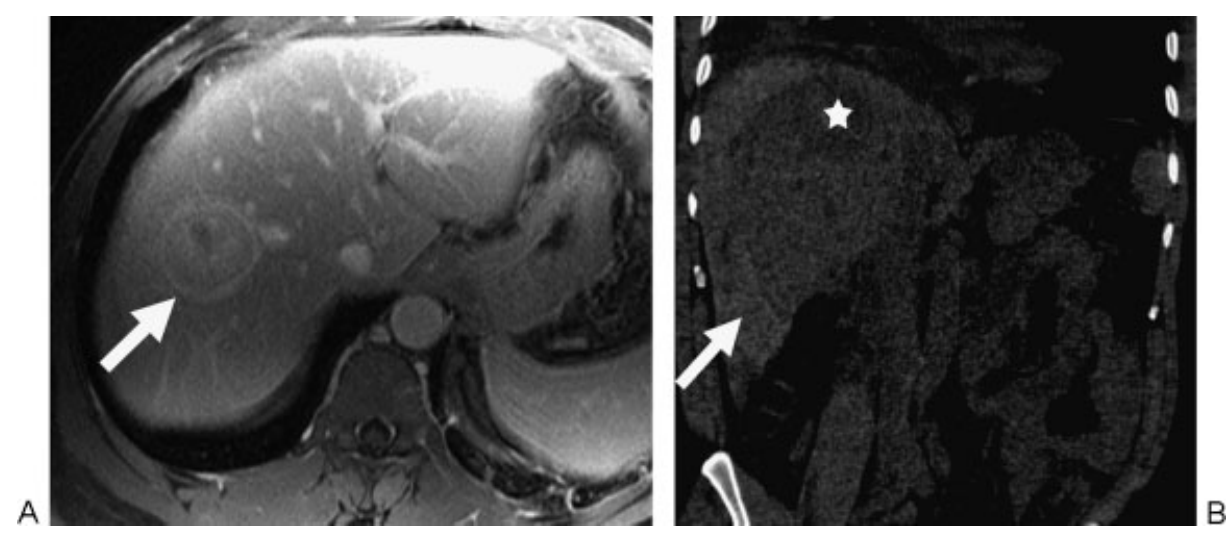

Figure 2 A 58-year-old man with hemophilia A and cirrhosis secondary to hepatitis C. (A) Axial T1 fat-saturated image postgadolinium demonstrates a 4.2-cm lesion (arrow) with a pseudocapsule that was a biopsy-proven hepatocellular carcinoma (HCC). (B) Coronal noncontrast computed tomography postablation, demonstrating an area of low attenuation at the ablation zone (star) and a subcapsular high attenuation hematoma (arrow). Two months postablation the patient underwent a liver transplant. 

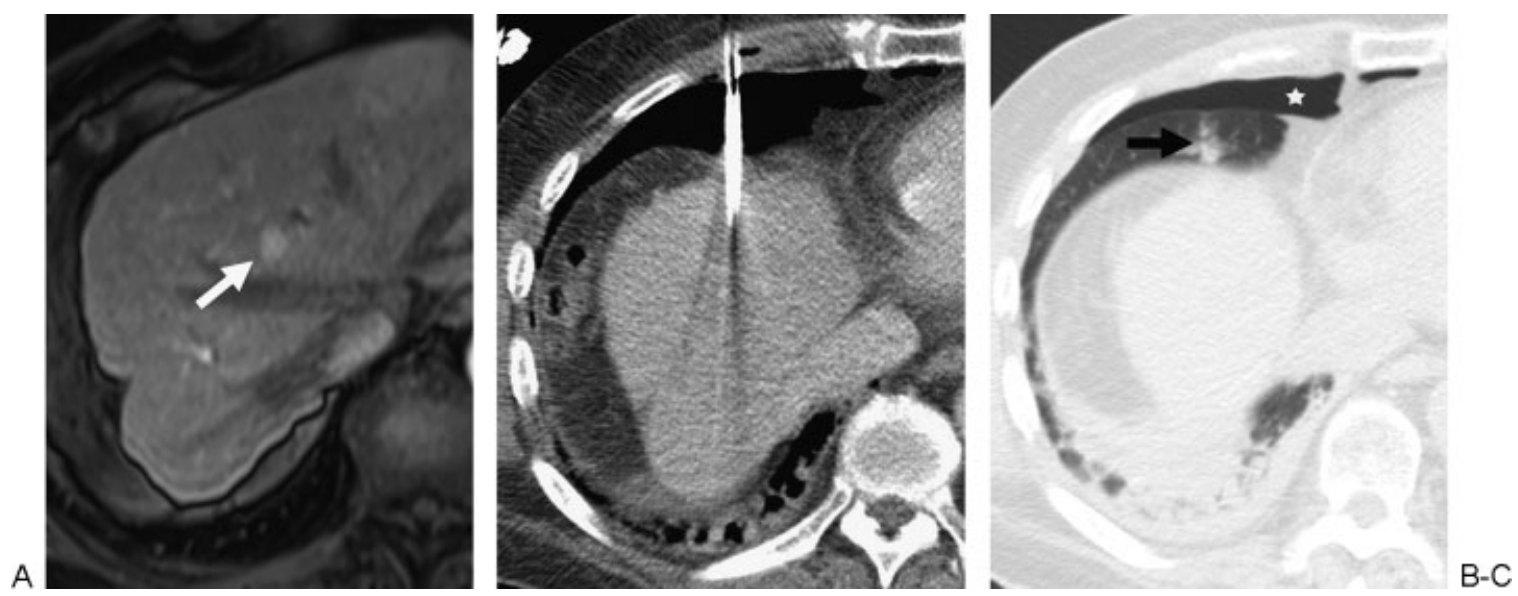

Figure 3 A 76-year-old man with cirrhosis secondary to hemochromatosis. (A) Axial T1 fat-saturated image postgadolinium demonstrates a 1-cm arterially enhancing lesion (arrow) that was a biopsy-proven hepatocellular carcinoma. (B) A computed tomography scan performed during the ablation procedure shows the cluster ablation electrode traversing the lung. (C) Postprocedure image demonstrates a pneumothorax (star). The track of the electrode through the lung is also seen (arrow). The patient required chest tube insertion because the pneumothorax increased in size on follow-up imaging.

that bilomas occurred in $3.3 \%$ of cases (109 of 3284) following RFA but only one required percutaneous drainage. ${ }^{6}$ One study demonstrated that intraoperative central bile duct cooling could prevent biliary complications in patients undergoing RFA for periductal HCC. ${ }^{7}$ Other studies have found that intraductal chilled saline perfusion through a nasobiliary tube was a potential intervention to prevent biliary injury by percutaneous RFA. ${ }^{8,9}$ These are small studies, and this practice is not yet widespread.

Koda et al found that a total of 113 extrahepatic organ injures occurred $(0.69 \%)$ including 2 patients with cardiac tamponade, 9 cases of pneumothorax (-Fig. 3) (0.06\%), 9 gastrointestinal injuries, 5 gallbladder injuries, 8 diaphragmatic injuries, 43 refractory pleural effusions, and 32 skin burns. ${ }^{5}$ Most of the extrahepatic complications due to thermal damage may be prevented by artificial ascites or pleural effusion, which creates a space between the tumor and adjacent organs such as the abdominal wall, diaphragm, gastrointestinal tract, or gallbladder ( - Fig. 4). ${ }^{10-12}$ Artificial ascites, however, poses a theoretical risk of a heat-sink effect that may decrease the effectiveness of the ablation. This risk was not proven in a study on percutaneous RFA of hepatic tumors abutting the diaphragm. ${ }^{13}$

Koda et al also found tumor seeding at the needle tract in seven cases $(0.04 \%)$ and eight cases of peritoneal dissemination $(0.05 \%),{ }^{5}$ which was significantly lower than the $12 \%$ reported in an earlier study. ${ }^{14} \mathrm{~A}$ recent literature review found that the risk of seeding was $0.61 \%$ ( 0 to $5.56 \%$ ) for RFA without biopsy and $0.95 \%$ (0 to $12.5 \%$ ) for RFA with biopsy. ${ }^{15}$ Subcapsular location and poor differentiation have been associated with an increased risk of seeding. ${ }^{14}$

Another major drawback of RFA is the high rate of disease recurrence after treatment (-Figs. $\mathbf{5}$ and $\mathbf{6}$ ). A few studies, predominantly from early experiences with RFA, found a statistically significantly shorter recurrence-free interval in patients with subcapsular tumors compared with nonsubcapsular tumors following RFA. ${ }^{16-18}$ However, more recent studies have found that the expected morbidity and local tumor progression in patients with subcapsular liver tumors who undergo percutaneous RFA was comparable with those in patients with nonsubcapsular tumors. ${ }^{19,20}$ Another early study found that the presence of vessels at least $3 \mathrm{~mm}$ in size contiguous to the hepatic tumors is a strong independent predictor of incomplete tumor destruction by RFA. ${ }^{21}$ Again, a more recent study found that RFA was an effective procedure, even for high-risk tumors adjacent to large blood vessels. ${ }^{22}$

\section{Follow-Up After Ablation}

Because tumor is treated in situ, imaging follow-up is necessary to assess treatment response. Protocols vary among institutions, but ongoing surveillance is performed with shorter intervals initially. At our institution, contrast-enhanced imaging with CT or MRI (with subtraction images) is performed at 1,3,6, 9, and 12 months after treatment and at 6-month intervals thereafter. On the initial follow-up imaging, successful ablation is seen as a nonenhancing area with or without an enhancing rim. The enhancing rim that may be observed along the periphery of the ablation zone appears to be a relatively concentric, symmetrical, and uniform process in an area with smooth inner margins and without nodularity (- Fig. 4). ${ }^{23}$ Benign peri-ablation enhancement must be differentiated from irregular peripheral enhancement due to residual tumor that occurs at the treatment margin. Ablation site recurrence can present as focal enhancing lesions within or around the ablation zone or as an overall increase in the size of the ablation zone. ${ }^{24}$

\section{Primary Liver Tumors}

\section{Hepatocellular Carcinoma}

HCC is well suited to treatment with locoregional therapy because it has a tendency to stay within the liver, with distant metastasis generally occurring late. RFA plays a role in 

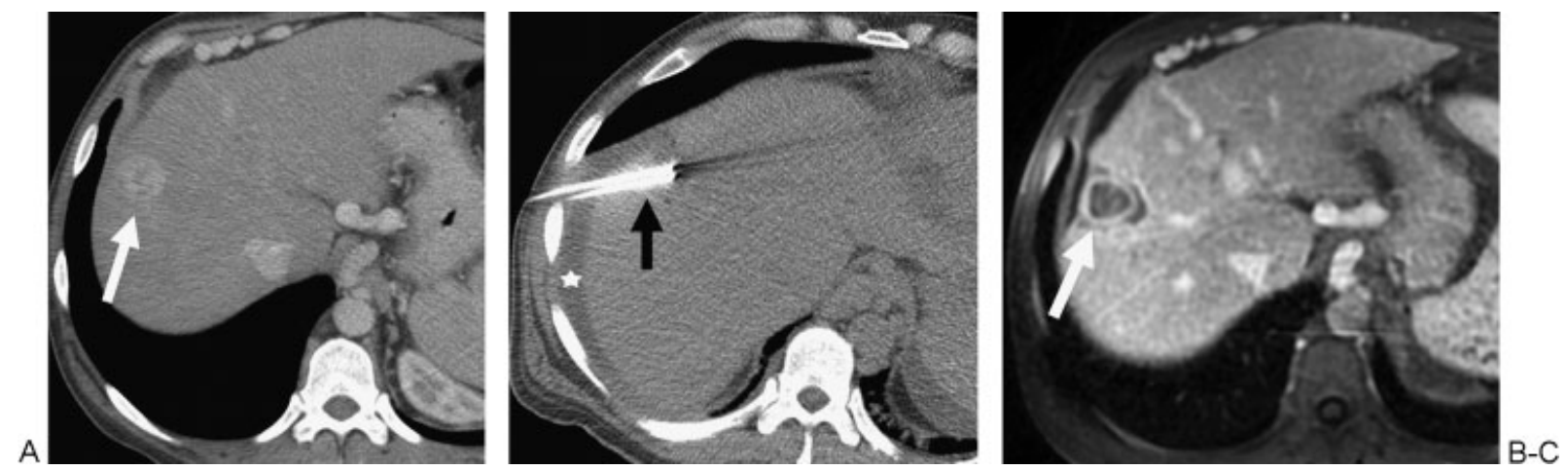

Figure 4 A 51-year-old man with cirrhosis secondary to hepatitis C. (A) Contrast-enhanced computed tomography demonstrates a 2.8-cm arterially enhancing lesion (arrow) consistent with a hepatocellular carcinoma (HCC). (B) During the procedure, hydrodissection was performed with $800 \mathrm{~mL}$ of $5 \%$ dextrose in water (star), which displaced the lung anteriorly allowing insertion of the electrode (arrow) without traversing the lung. (C) T1 fat-saturated magnetic resonance image postgadolinium 3 months postablation demonstrates a thin enhancing rim without nodularity (arrow), compatible with a complete ablation.

multiple situations: resectable HCC, unresectable HCC, as a bridge to transplant, and in recurrent HCC.

\section{Resectable HCC}

Prior to the routine use of liver transplant, surgical resection was the only modality for curative treatment of HCC. Based on the Barcelona-Clinic Liver Cancer (BCLC) treatment strategy, resection is considered the first treatment option for earlystage patients (single tumor $<2 \mathrm{~cm}$ ). ${ }^{25}$ A recent study, however, reported that the efficacy and safety of percutaneous RFA were better than those of surgical resection with HCC measuring $\leq 2 \mathrm{~cm}$, especially those with central $\mathrm{HCC}^{26} \mathrm{~A}$ study comparing resection or RFA in patients with a solitary HCC $<5 \mathrm{~cm}$ reported 1 - and 4-year overall survival rates after percutaneous RFA and surgery of $96 \%, 68 \%$, and $93 \%, 64 \%$, respectively. ${ }^{27}$ The corresponding disease-free survival rates were $86 \%, 46 \%$, and $87 \%, 52 \%$, respectively. ${ }^{27}$ A randomized trail comparing RFA and surgical resection for HCC conforming to the Milan criteria reported a 1-, 2-, 3-, 4- and 5-year overall survival rates for the RFA group and the resection group of $87 \%, 77 \%, 70 \%, 66 \%, 55 \%$, and $98 \%, 97 \%, 92 \%, 83 \%, 76 \%$, respectively. ${ }^{28}$ The 1-, 2-, 3-, 4-, and 5-year overall recurrence rates were $17 \%, 38 \%, 50 \%, 59 \%$, and $63 \%$ for the RFA group and $12 \%, 23 \%, 34 \%, 39 \%$, and $42 \%$ for the resection group. ${ }^{28}$

\section{Unresectable HCC}

A retrospective study comparing the outcome of RFA and trans-arterial chemoembolization (TACE) in patients with unresectable HCC found that the 1- and 2-year overall survival rates were $82 \%$ and $72 \%$, respectively, in the RFA group and $80 \%$ and $58 \%$, respectively, in the TACE group. ${ }^{29} \mathrm{~A}$ second study found that the overall recurrence-free rate was significantly higher in the RFA group than in the TACE group: The 2-year local recurrence-free rates in the RFA and TACE groups were $60 \%$ and $49 \%$, respectively. ${ }^{30}$ A meta-analysis of five randomized controlled trials comparing RFA and percutaneous ethanol injection (PEI) demonstrated that patients treated with RFA had better 1- and 3-year overall survival
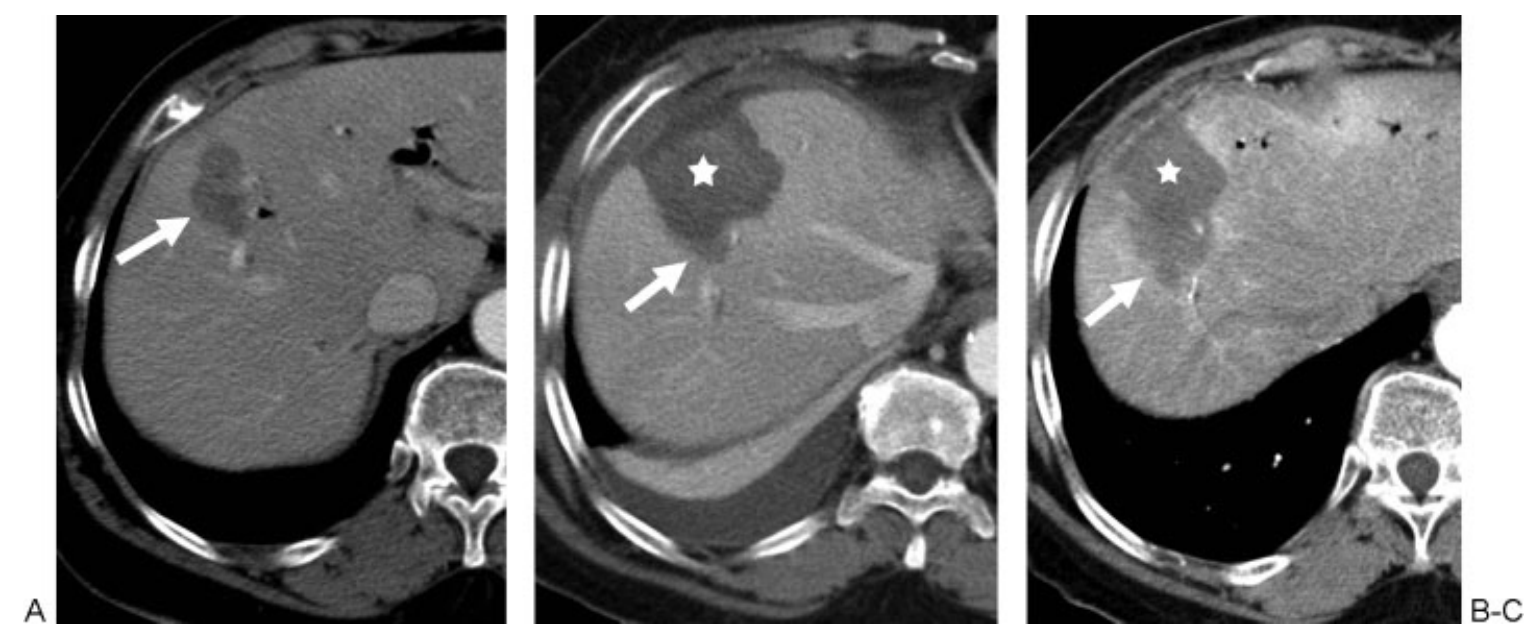

Figure 5 A 72-year-old man with colorectal carcinoma. (A) Postcontrast computed tomography (CT) demonstrates a 3-cm hypoattenuating lesion (arrow) consistent with a metastasis. (B) Postcontrast CT 1-month postablation demonstrates a low attenuation ablation zone (star) with a focus of residual tumor posteriorly (arrow). (C) Postcontrast CT 6-months postablation shows the ablation zone (star) and that the focus of residual tumor has increased in size (arrow). 

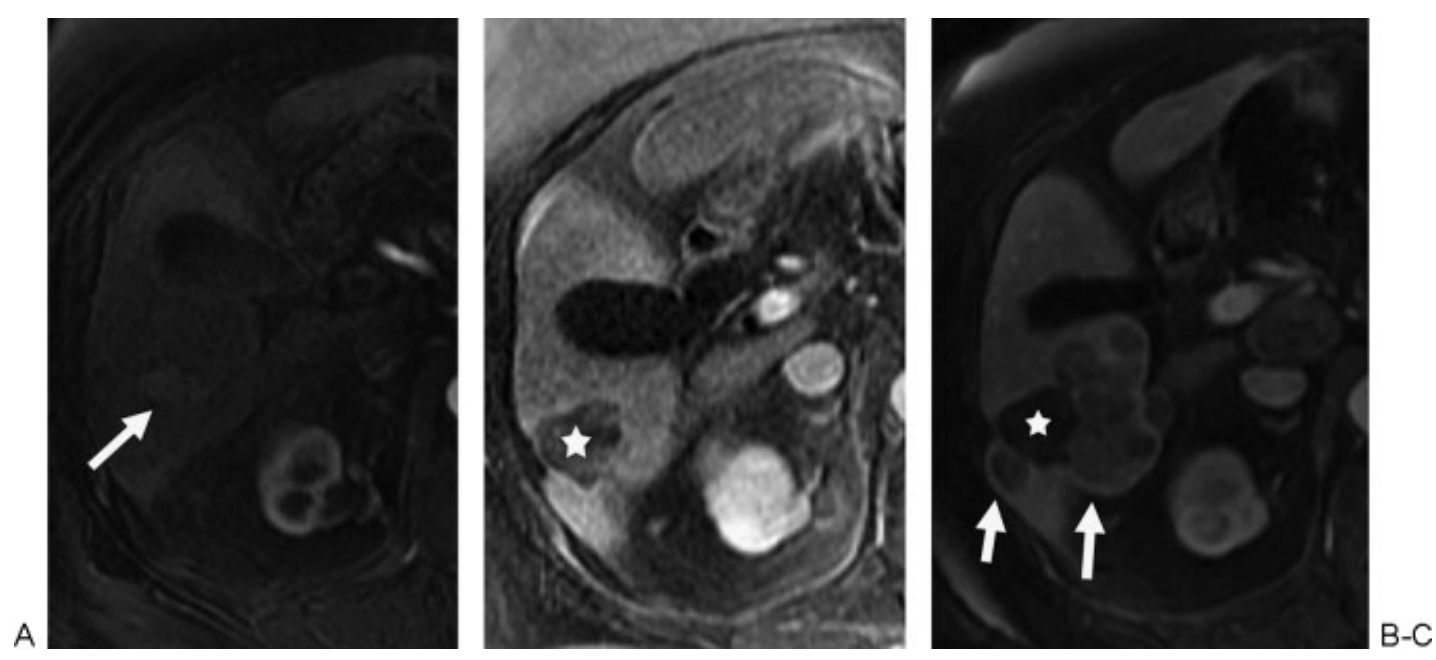

Figure 6 A 66-year-old man with cirrhosis secondary to alcohol dependency. (A) T1 fat-saturated image postgadolinium demonstrates a 3-cm arterially enhancing lesion (arrow). (B) Postgadolinium image 9 months postablation shows an ablation zone (arrow) without evidence of residual disease. (C) Postgadolinium image 2 years postablation demonstrates multiple areas of washout on delayed images (arrows) adjacent to the ablation zone (star), consistent with recurrent disease.

rates than those treated with PEI. ${ }^{31}$ Disease recurrence rates at the ablation site were also significantly lower in the RFA group than in the PEI group (2 to $14 \%$ versus 11 to $35 \%$ ). ${ }^{31}$ The combination of RFA with other locoregional treatments such as PEI or TACE has been shown to result in better survival and lower recurrence rates than RFA alone. ${ }^{32-34}$

\section{Bridge to Transplant}

The concept of liver transplant as the treatment of HCC is evolving. According to the Milan criteria, patients with a large single $(\leq 5 \mathrm{~cm})$ or multiple tumors (fewer than three and $\leq 3 \mathrm{~cm}$ in size) are eligible for transplantation (-Fig. 2). However, because of the discrepancy between the increasing demand and the inadequate supply of liver from deceased donors, many potential recipients either die before an organ becomes available or drop off the transplant waiting list because of tumor progression. RFA and other locoregional treatments, such as TACE and PEI, have an emerging role to play as bridging interventions. Using the UNOS inclusion criteria, a study found that the respective dropout probabilities at 6,12 , and 18 months would have been $11.0 \%, 57.4 \%$, and $68.7 \%$, respectively. ${ }^{35}$ In comparison, a study that used multimodality ablative techniques, such as RFA, TACE, ethyl alcohol ablation, or a combination of techniques as a bridge for transplantation, found a dropout rate of $0 \%, 0 \%$, and $6 \%$ at 6,12 , and 24 months using the same exclusion criteria. ${ }^{36}$ The role of RFA alone as a bridge to transplantation was specifically addressed in two studies. One study reported a dropout rate of $14 \%$ after a waiting time of 11.9 months, ${ }^{37}$ and the other a rate of $5.8 \%$ after a mean waiting time of 12.7 months. ${ }^{38}$

\section{Recurrent HCC}

Recurrent HCC occurs in 50 to $80 \%$ of patients at 5 years after resection, with the majority occurring within 2 years after resection. ${ }^{39}$ RFA has been increasingly used in these patients, with cohort studies reporting the 5-year overall survival rate ranging from 18 to $52 \%{ }^{39}$

\section{Intrahepatic Cholangiocarcinoma}

Hepatic resection is the only curative treatment option for intrahepatic cholangiocarcinoma; however, most patients are not candidates for this option because of either advanced disease at the time of presentation, likely insufficient function of the remaining portion of the liver, or poor surgical candidacy. Patients with untreated unresectable cholangiocarcinoma have a median survival of 3.9 months. ${ }^{40} \mathrm{~A}$ recent study reported a median overall survival period of 38.5 months in patients with unresectable primary intrahepatic cholangiocarcinoma treated with RFA. ${ }^{41}$ This is likely highly influenced by patient selection with tumors small enough in both size and number to be treated with RFA.

\section{Metastatic Disease}

Over the last 2 decades, advances in surgical techniques have led to effective treatment for selected patients with hepatic metastases. However, only 10 to $25 \%$ of patients with metastases isolated to the liver are eligible for resection because of extent and location of disease or concurrent medical conditions. ${ }^{1}$ RFA has proved to be a safe and feasible treatment option for extending survival in select patients with liver metastases from many different primaries such as colorectal cancer (- Fig. 5), ${ }^{42,43}$ pancreatic cancer, ${ }^{44}$ cholangiocarcinoma ${ }^{45}$ neuroendocrine tumors, ${ }^{46}$ breast cancer, ${ }^{47}$ and gastric cancer. ${ }^{48}$

Studies on the long-term survival of nonsurgical patients with hepatic colorectal metastases who underwent RFA reported a 1-year survival rate of 86 to $99 \%$, a 3-year survival rate of 46 to $68 \%$, and a 5 -year survival rate of 24 to $44 \%{ }^{1}$ Another study found that the 3-year survival rate in patients with a solitary colorectal metastases was $55 \%$ for patients treated with surgery and $52 \%$ for those who underwent RFA. ${ }^{49}$ A more recent study reported that the overall survival and disease-free survival did not differ between patients treated with resection or RFA in patients with a solitary 
colorectal metastasis $<3 \mathrm{~cm}$; however, in patients with a solitary metastasis $>3 \mathrm{~cm}$, the disease-free survival was significantly lower in the RFA group as compared with the resection group. ${ }^{42} \mathrm{~A}$ recently published systematic review, however, concluded that the available evidence was insufficient to recommend RFA for a radical oncologic treatment of colorectal liver metastases. ${ }^{50}$

\section{Conclusion}

RFA has been shown to achieve effective and reproducible local tumor control with minimal morbidity in patients with small HCC and metastases. It is a desirable treatment option in patients with early-stage HCC when resection or transplant is precluded or delayed. It has also been proven a viable treatment option in patients with limited hepatic metastatic disease. For large tumor burdens, RFA is limited in effectiveness, and alternatives such as TACE or systemic therapies are preferred.

\section{References}

1 Lencioni R, Crocetti L. Radiofrequency ablation of liver cancer. Tech Vasc Interv Radiol 2007;10(1):38-46

2 Rhim H, Goldberg SN, Dodd GD III, et al. Essential techniques for successful radio-frequency thermal ablation of malignant hepatic tumors. Radiographics 2001;21(Spec No):S17-S35; discussion S36-S39

3 Goldberg SN, Gazelle GS, Mueller PR. Thermal ablation therapy for focal malignancy: a unified approach to underlying principles, techniques, and diagnostic imaging guidance. AJR Am J Roentgenol 2000;174(2):323-331

4 Dodd GD III, Frank MS, Aribandi M, Chopra S, Chintapalli KN. Radiofrequency thermal ablation: computer analysis of the size of the thermal injury created by overlapping ablations. AJR Am J Roentgenol 2001;177(4):777-782

5 Koda M, Murawaki Y, Hirooka Y, et al. Complications of radiofrequency ablation for hepatocellular carcinoma in a multicenter study: an analysis of 16346 treated nodules in 13,283 patients. Hepatol Res 2012;42(11):1058-1064

6 Chang IS, Rhim H, Kim SH, et al. Biloma formation after radiofrequency ablation of hepatocellular carcinoma: incidence, imaging features, and clinical significance. AJR Am J Roentgenol 2010; 195(5):1131-1136

7 Lam VW, Ng KK, Chok KS, et al. Safety and efficacy of radiofrequency ablation for periductal hepatocellular carcinoma with intraductal cooling of the central bile duct. J Am Coll Surg 2008; 207(2):e1-e5

8 Ohnishi T, Yasuda I, Nishigaki Y, et al. Intraductal chilled saline perfusion to prevent bile duct injury during percutaneous radiofrequency ablation for hepatocellular carcinoma. J Gastroenterol Hepatol 2008;23(8 Pt 2):e410-e415

9 Ogawa T, Kawamoto H, Kobayashi Y, et al. Prevention of biliary complication in radiofrequency ablation for hepatocellular carcinoma-cooling effect by endoscopic nasobiliary drainage tube. Eur J Radiol 2010;73(2):385-390

10 Song I, Rhim H, Lim HK, Kim YS, Choi D. Percutaneous radiofrequency ablation of hepatocellular carcinoma abutting the diaphragm and gastrointestinal tracts with the use of artificial ascites: safety and technical efficacy in 143 patients. Eur Radiol 2009;19(11):2630-2640

11 Kondo Y, Yoshida H, Shiina S, Tateishi R, Teratani T, Omata M. Artificial ascites technique for percutaneous radiofrequency ablation of liver cancer adjacent to the gastrointestinal tract. Br J Surg 2006;93(10):1277-1282
12 Iwai S, Sakaguchi H, Fujii H, et al. Benefits of artificially induced pleural effusion and/or ascites for percutaneous radiofrequency ablation of hepatocellular carcinoma located on the liver surface and in the hepatic dome. Hepatogastroenterology 2012;59 (114):546-550

13 Nam SY, Rhim H, Kang TW, et al. Percutaneous radiofrequency ablation for hepatic tumors abutting the diaphragm: clinical assessment of the heat-sink effect of artificial ascites. AJR Am J Roentgenol 2010;194(2):W227-W231

14 Llovet JM, Vilana R, Brú C, et al; Barcelona Clínic Liver Cancer (BCLC) Group. Increased risk of tumor seeding after percutaneous radiofrequency ablation for single hepatocellular carcinoma. Hepatology 2001;33(5):1124-1129

15 Stigliano R, Marelli L, Yu D, Davies N, Patch D, Burroughs AK Seeding following percutaneous diagnostic and therapeutic approaches for hepatocellular carcinoma. What is the risk and the outcome? Seeding risk for percutaneous approach of HCC. Cancer Treat Rev 2007;33(5):437-447

16 Komorizono Y, Oketani M, Sako K, et al. Risk factors for local recurrence of small hepatocellular carcinoma tumors after a single session, single application of percutaneous radiofrequency ablation. Cancer 2003;97(5):1253-1262

17 Hori T, Nagata K, Hasuike S, et al. Risk factors for the local recurrence of hepatocellular carcinoma after a single session of percutaneous radiofrequency ablation. J Gastroenterol 2003;38 (10):977-981

18 Kang TW, Rhim H, Kim EY, et al. Percutaneous radiofrequency ablation for the hepatocellular carcinoma abutting the diaphragm: assessment of safety and therapeutic efficacy. Korean J Radiol 2009;10(1):34-42

19 Sartori S, Tombesi P, Macario F, et al. Subcapsular liver tumors treated with percutaneous radiofrequency ablation: a prospective comparison with nonsubcapsular liver tumors for safety and effectiveness. Radiology 2008;248(2):670-679

20 Cho YK, Rhim H, Ahn YS, Kim MY, Lim HK. Percutaneous radiofrequency ablation therapy of hepatocellular carcinoma using multitined expandable electrodes: comparison of subcapsular and nonsubcapsular tumors. AJR Am J Roentgenol 2006; 186(5, Suppl):S269-S274

21 Lu DS, Raman SS, Limanond P, et al. Influence of large peritumoral vessels on outcome of radiofrequency ablation of liver tumors. J Vasc Interv Radiol 2003;14(10):1267-1274

22 Thanos L, Mylona S, Galani P, Pomoni M, Pomoni A, Koskinas I. Overcoming the heat-sink phenomenon: successful radiofrequency thermal ablation of liver tumors in contact with blood vessels. Diagn Interv Radiol 2008;14(1):51-56

23 Crocetti L, de Baere T, Lencioni R. Quality improvement guidelines for radiofrequency ablation of liver tumours. Cardiovasc Intervent Radiol 2010;33(1):11-17

24 Kele PG, de Jong KP, van der Jagt EJ. Increase in volume of ablation zones during follow-up is highly suggestive of ablation site recurrence in colorectal liver metastases treated with radiofrequency ablation. J Vasc Interv Radiol 2012;23(4):537-544

25 Llovet JM, Fuster J, Bruix J; Barcelona-Clínic Liver Cancer Group. The Barcelona approach: diagnosis, staging, and treatment of hepatocellular carcinoma. Liver Transpl 2004;10(2, Suppl 1): S115-S120

26 Peng ZW, Lin XJ, Zhang YJ, et al. Radiofrequency ablation versus hepatic resection for the treatment of hepatocellular carcinomas $2 \mathrm{~cm}$ or smaller: a retrospective comparative study. Radiology 2012;262(3):1022-1033

27 Chen MS, Li JQ, Zheng Y, et al. A prospective randomized trial comparing percutaneous local ablative therapy and partial hepatectomy for small hepatocellular carcinoma. Ann Surg 2006;243 (3):321-328

28 Huang J, Yan L, Cheng Z, et al. A randomized trial comparing radiofrequency ablation and surgical resection for HCC conforming to the Milan criteria. Ann Surg 2010;252(6):903-912 
29 Chok KS, Ng KK, Poon RT, et al. Comparable survival in patients with unresectable hepatocellular carcinoma treated by radiofrequency ablation or transarterial chemoembolization. Arch Surg 2006;141(12):1231-1236

30 Murakami T, Ishimaru H, Sakamoto I, et al. Percutaneous radiofrequency ablation and transcatheter arterial chemoembolization for hypervascular hepatocellular carcinoma: rate and risk factors for local recurrence. Cardiovasc Intervent Radiol 2007;30(4):696-704

31 Tiong L, Maddern GJ. Systematic review and meta-analysis of survival and disease recurrence after radiofrequency ablation for hepatocellular carcinoma. Br J Surg 2011;98(9):1210-1224

32 Zhang YJ, Liang HH, Chen MS, et al. Hepatocellular carcinoma treated with radiofrequency ablation with or without ethanol injection: a prospective randomized trial. Radiology 2007;244 (2):599-607

33 Yang P, Liang M, Zhang Y, Shen B. Clinical application of a combination therapy of lentinan, multi-electrode RFA and TACE in HCC. Adv Ther 2008;25(8):787-794

34 Morimoto M, Numata K, Kondou M, Nozaki A, Morita S, Tanaka K. Midterm outcomes in patients with intermediate-sized hepatocellular carcinoma: a randomized controlled trial for determining the efficacy of radiofrequency ablation combined with transcatheter arterial chemoembolization. Cancer 2010;116(23):5452-5460

35 Yao FY, Bass NM, Nikolai B, et al. A follow-up analysis of the pattern and predictors of dropout from the waiting list for liver transplantation in patients with hepatocellular carcinoma: implications for the current organ allocation policy. Liver Transpl 2003;9(7): 684-692

36 Fisher RA, Maluf D, Cotterell AH, et al. Non-resective ablation therapy for hepatocellular carcinoma: effectiveness measured by intention-to-treat and dropout from liver transplant waiting list. Clin Transplant 2004;18(5):502-512

37 Brillet PY, Paradis V, Brancatelli G, et al. Percutaneous radiofrequency ablation for hepatocellular carcinoma before liver transplantation: a prospective study with histopathologic comparison. AJR Am J Roentgenol 2006;186(5, Suppl):S296-S305

38 Lu DS, Yu NC, Raman SS, et al. Percutaneous radiofrequency ablation of hepatocellular carcinoma as a bridge to liver transplantation. Hepatology 2005;41(5):1130-1137
39 Lau WY, Lai EC. The current role of radiofrequency ablation in the management of hepatocellular carcinoma: a systematic review. Ann Surg 2009;249(1):20-25

40 Park J, Kim MH, Kim KP, et al. Natural history and prognostic factors of advanced cholangiocarcinoma without surgery, chemotherapy, or radiotherapy: a large-scale observational study. Gut Liver 2009;3(4):298-305

$41 \mathrm{Kim}$ JH, Won HJ, Shin YM, Kim KA, Kim PN. Radiofrequency ablation for the treatment of primary intrahepatic cholangiocarcinoma. AJR Am J Roentgenol 2011;196(2):W205-9

$42 \mathrm{Kim} \mathrm{KH}$, Yoon YS, Yu CS, et al. Comparative analysis of radiofrequency ablation and surgical resection for colorectal liver metastases. J Korean Surg Soc 2011;81(1):25-34

43 Van Tilborg AA, Meijerink MR, Sietses C, et al. Long-term results of radiofrequency ablation for unresectable colorectal liver metastases: a potentially curative intervention. Br J Radiol 2011;84(1002): 556-565

44 Park JB, Kim YH, Kim J, et al. Radiofrequency ablation of liver metastasis in patients with locally controlled pancreatic ductal adenocarcinoma. J Vasc Interv Radiol 2012;23(5):635-641

45 Park SY, Kim JH, Won HJ, Shin YM, Kim PN. Radiofrequency ablation of hepatic metastases after curative resection of extrahepatic cholangiocarcinoma. AJR Am J Roentgenol 2011;197(6): W1129-W1134

46 Gamblin TC, Christians K, Pappas SG. Radiofrequency ablation of neuroendocrine hepatic metastasis. Surg Oncol Clin N Am 2011;20 (2):273-279, vii-viii

47 Illing R, Gillams A. Radiofrequency ablation in the treatment of breast cancer liver metastases. Clin Oncol (R Coll Radiol) 2010;22 (9):781-784

$48 \mathrm{Kim} \mathrm{HR}$, Cheon SH, Lee KH, et al. Efficacy and feasibility of radiofrequency ablation for liver metastases from gastric adenocarcinoma. Int J Hyperthermia 2010;26(4):305-315

49 Oshowo A, Gillams A, Harrison E, Lees WR, Taylor I. Comparison of resection and radiofrequency ablation for treatment of solitary colorectal liver metastases. Br J Surg 2003;90(10):1240-1243

50 Cirocchi R, Trastulli S, Boselli C, et al. Radiofrequency ablation in the treatment of liver metastases from colorectal cancer. Cochrane Database Syst Rev 2012;6:CD006317 